\title{
Selective ligation using a detachable snare for small-intestinal polyps in patients with Peutz-Jeghers syndrome
}

Peutz-Jeghers syndrome (PJS) is a rare autosomal dominant hereditary disorder with an incidence of $1 / 200000$ live births. It is partly characterized by hamartomatous polyps of the gastrointestinal tract. The risk of malignant transformation in these polyps increases with polyp size, with cancer occurring in $15 \%$ of polyps more than $3 \mathrm{~cm}$ in diameter [1]. Hamartomatous polyps are detected in $88 \%$ of patients with PJS, mostly in the small intestine. We evaluated the therapeutic efficacy, clinical impact, and safety of selective ligation using a detachable snare for small-intestinal polyps in three patients with PJS. For larger polyps, this technique is safer than conventional snare polypectomy or endoscopic mucosal resection (EMR) because it minimizes the risks of postpolypectomy bleeding, thermal injury to deeper tissue layers, and perforation.

A 38-year-old man diagnosed with PJS at the age of 9 years with a history of endoscopic polypectomy, laparotomy, and partial small-bowel resection underwent barium-contrast radiography, which revealed that multiple small-intestinal polyps remained. He was hospitalized, and single-balloon enteroscopy (SBE) was first performed via the oral approach, during which the small intestine was examined for over $180 \mathrm{~cm}$ from the ligament of Treitz. Small polyps were removed with conventional snare polypectomy or EMR. Three large pedunculated polyps up to a maximum diameter of $15 \mathrm{~mm}$ were treated by a technique that used a detachable snare (Ligating device; Olympus, Tokyo, Japan; Fig. 1). The furthest point reached during the endoscopy was marked by submucosal injection of ink.

After 4 months, the SBE was repeated via the oral approach in order to assess the post-treatment lesions. The location of the marking point was confirmed and all post-treatment lesions were either identified as clean without an obvious ulcer scar or were not clearly visualized due to being covered by the villi of the small intestine. No complications were reported either during or after the SBE procedure and polypectomy using the detachable snare

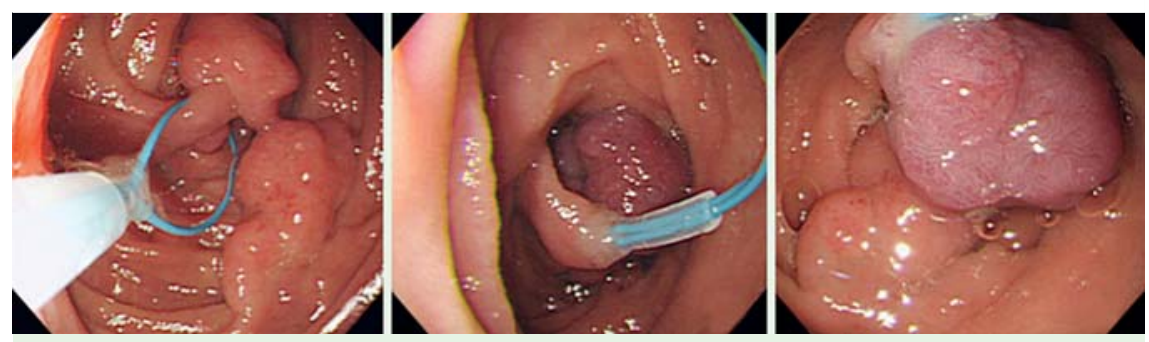

Fig. 1 The polypectomy procedure using the detachable snare. a The detachable snare is placed over the neck of the polyp stalk. $\mathbf{b}$ The snare is tightened. $\mathbf{c}$ The polyp becomes paler in color because of ischemia due to the tightened detached snare.

in this patient or in two other patients who underwent the same technique.

Balloon enteroscopy is a recently developed procedure for the investigation of small-intestinal disorders, which is expected to be well suited to the diagnosis and treatment of small-intestinal polyps in patients with PJS, and may reduce the incidence of obstruction and the consequent need for multiple surgical procedures. Such repeated interventions can potentially lead to serious adhesions and short bowel syndrome. Ross et al. reported that laparoscopy-assisted double-balloon enteroscopy (DBE) held promise as a new technique for small-intestinal polyps in patients with PJS [2]. Akahoshi et al. reported that the newly developed grasping-type scissors forceps were useful for endoscopic resection of a large pedunculated duodenal polyp diagnosed as a Peutz-Jeghers-type polyp [3]. Initial studies have reported that the major complication of endoscopic polypectomy is bleeding with an estimated incidence of about $2 \%$, more frequently associated with the removal of large pedunculated polyps [4].

The main advantage of the detachable snare technique is the decreased risk of bleeding and perforation. It may result in complete healing of polyps or shrink larger polyps when the aim is not to completely eradicate them. Compared with the conventional endoscopic technique, it may also decrease the risk of hemorrhage and perforation if the polyp grows.

The technique was used for polyps with stalk sizes of more than $10 \mathrm{~mm}$ in diameter, whilst conventional polypectomy was performed on polyps with diameters less than $10 \mathrm{~mm}$. Polyps with diameters less than $5 \mathrm{~mm}$ were left untreated because typically there are so many of them that it would take too long to reach the larger polyps. Polyps with diameters exceeding $30 \mathrm{~mm}$ or with findings suspicious of malignancy on endoscopic or radiographic investigations such as computed tomography $(\mathrm{CT})$ and magnetic resonance imaging (MRI) were treated surgically because they were too large to be handled endoscopically and had the potential to be malignant. A total of eight polyps with an average diameter of $21.9 \mathrm{~mm}$ were treated using the detachable snare technique. The use of the detachable snare makes it easier to obtain the optimal tightness, as the head of the polyp becomes paler in color immediately following ligation (৫ Fig. 1). The average duration of hospitalization was 9 days.

This study has some limitations because tissue samples of the snared polyps could not be examined, and therefore histopathological diagnoses could not be made. In two of the three patients, a comprehensive exploration of the small intestine could not be achieved so curable polyps could not be reached, because of severe intra-abdominal adhesions due to previous extensive abdominal surgery. In conclusion, selective ligation with a detachable snare is a useful alternative method for the treatment of large polyps in the small intestines of patients with PJS, especially in those with a history of extensive abdominal surgery. 
Endoscopy_UCTN_Code_TTT_1AP_2AD

Competing interests: None

K. Takakura ${ }^{1}$, T. Kato ${ }^{2}$, S. Arihiro ${ }^{1}$, T. Miyazaki ${ }^{1}$, Y. Arai ${ }^{1}$, Y. Nakao ${ }^{1}$,

N. Komoike ${ }^{1}$, M. Itagaki ${ }^{1}$, I. Odagi ${ }^{1}$, K. Hirohama ${ }^{1}$, M. Saruta ${ }^{1}$, M. Matsuoka ${ }^{1}$, H. Tajiri ${ }^{1,2}$

1 Division of Gastroenterology and Hepatology, Department of Internal Medicine, The Jikei University School of Medicine, Tokyo, Japan

2 Department of Endoscopy, The Jikei University School of Medicine, Tokyo, Japan

\section{References}

1 Spigelman AD, Murday V, Phillips RK. Cancer and the Peutz-Jeghers syndrome. Gut 1989; 30: $1588-1589$

2 Ross A, Dye C, Prachand V. Laparoscopic-assisted double-balloon enteroscopy for small-bowel polyp surveillance and treatment in patients with Peutz-Jeghers syndrome. Gastrointest Endosc 2006; 64: 984 988

3 Akahoshi K, Honda K, Kubokawa M et al. Endoscopic resection of a large pedunculated duodenal polyp using a grasping type scissors forceps. Endoscopy 2008; 40: E74 - E75

4 Brandimarte G, Tursi A. Endoscopic snare excision of large pedunculated colorectal polyps: a new, safe, and effective technique. Endoscopy 2001; 33: 854-857
Bibliography

DOI $10.1055 / \mathrm{s}-0030-1256528$

Endoscopy 2011; 43: E264 -E265

(c) Georg Thieme Verlag KG Stuttgart · New York . ISSN 0013-726X

Corresponding author

T. Kato, MD, PhD

Department of Endoscopy

The Jikei University School of Medicine 3-25-8 Nishi-shimbashi

Minato-ku

Tokyo 105-8461

Japan

Fax: +81-3-5443-3228

tkato@jikei.ac.jp 